\title{
Formation and Propagation of Local Traffic Jam
}

\author{
Hong-sheng Qi, ${ }^{1}$ Dian-hai Wang, ${ }^{1}$ and Peng Chen ${ }^{2}$ \\ ${ }^{1}$ College of Civil Engineering Architecture, Zhejiang University, 866, Yuhngtang Road, Hangzhou City, \\ Zhejiang Province 310058, China \\ ${ }^{2}$ Department of Civil Engineering, Nagoya University, Furo-cho, Chikusa-ku, Nagoya, Japan
}

Correspondence should be addressed to Hong-sheng Qi; lordqi@yahoo.cn

Received 14 November 2012; Accepted 22 January 2013

Academic Editor: Cengiz Çinar

Copyright (c) 2013 Hong-sheng Qi et al. This is an open access article distributed under the Creative Commons Attribution License, which permits unrestricted use, distribution, and reproduction in any medium, provided the original work is properly cited.

\begin{abstract}
Large scale traffic congestion often stems from local traffic jam in single road or intersection. In this paper, macroscopic method was used to explore the formation and propagation of local traffic jam. It is found that (1) the propagation of traffic jam can be seen as the propagation of traffic signal parameters, that is, virtual split and virtual green time; (2) for a road with endogenous flow, entrance location influences the jam propagation. With the same demand (upstream links flow and entrance flow), the upstream got more influence; (3) when a one-lane road is thoroughly congested, virtual signal parameters everywhere are the same as that at stop line; for a basic road, the virtual signals work in a cooperative manner; (4) phase sequence is one important parameter that influences traffic performances during peak hour where spill back of channelization takes place. The same phase plan for left-turn flow and through flow would be preferred; (5) signal coordination plays an important role in traffic jam propagation and hence effective network signal parameters should be designed to prevent jam from propagation to the whole network. These findings would serve as a basis for future network traffic congestion control.
\end{abstract}

\section{Introduction}

Urban road network has been considered as the "life line" of urban daily life. It serves as the basis of urban economy. The assurance of its normal operation is very important. However, as the development of urbanization, the number of vehicles relatively exceeds the capacity of road network which often results in large scale traffic jam during peak hours. This leads to many problems such as pollution, and noise. Large scale traffic jam often stems from local jam which takes place in a road or single node. According to Wright and Roberg [1], there are three sources of traffic jam: a temporary obstruction, stochastic fluctuation in demand, and permanent capacity bottleneck. These types of congestion cannot be prevented efficiently because they are related tightly with road geometry, land usage, or other factors which cannot be changed easily. However, if we can control the propagation of traffic jam, then, network traffic jam will be prevented. The propagation process looks like "domino phenomena" which is characterized by connected set of events. Many literatures are focusing on this subject.
Simulation methods including microscopic and macroscopic types are among the most used methods. Microscopic tools include cellular automata [2] and car following models [3]. They are based on dynamic motion of single vehicle and hence can grasp the traffic performance of the whole network $[1,4,5]$; macroscopic tools often adopted a discrete form $[6,7]$ of LWR model which stemmed from Lighthill and Whitham [8] and Richards [9]. This method divided links into a series of consecutive cells and time into equal time steps. By checking and tracking basic traffic parameters (flow, density, and velocity) of every cell during each step, the dynamic nature of traffic jam can be derived. This kind of method greatly relies on underlying models and the simulation networks are often regular $[10,11]$ which lacks generality. Furthermore, no analytical conclusion can be obtained through this approach.

Some researchers tried to establish analytical results of traffic jam. Wright and Roberg [1] proposed a simple analytical model for incident-based jam growth and discussed the effect of the length of the channelized part of roads and stop line width assignment on jam formation; Michalopoulos 


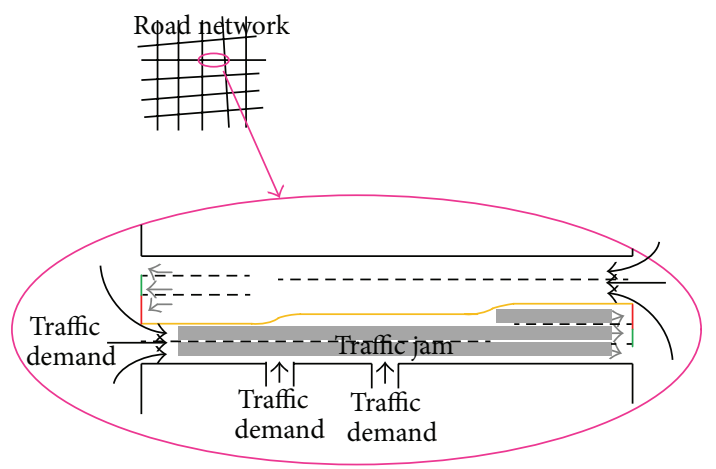

FIGURE 1: Simple representation of road geometry.

et al. [12] formulated a rigorous but complex mathematical modeling for signalized links; Skabardonis and Geroliminis $[13,14]$ developed an analytical model based on kinetic wave theory to construct monitor methods for arterials. Based on the derivation of waves produced by traffic signal, vehicle trajectory was identified and hence travel time function was formulated; Daganzo [15] divided the network into connected neighborhoods and constructed the relationship between defined traffic variables and formulated the so-called "gridlock" condition. Through these researches we can get a deep understanding of traffic jam. If we examine the general road network, we can see that it is comprised of basic road (some may be simpler) as shown in Figure 1. The road is multilane structure controlled by traffic signal. The traffic demand may come from upstream links or entrance within roads. Compared with this situation, current researches are not enough to describe traffic flow operation on basic road segment, not to mention the network traffic jam propagation.

Some scholars used data collected from control system (mainly loop detectors) to monitor long queue and spillover $[16,17]$. However, under congestion hour traffic jam involves various traffic flows that include multilane flow and endogenous flow from within the entrance, and these are difficult to distinguish from loop detector data. These factors may undermine the applicability of these methods.

The dynamic nature of network traffic jam (critical condition for its formation, propagation speed, propagation route, and so on) is complex in that they are influenced by many factors such as road physical characteristics, network topology, and control parameters. Understanding of these relationships is important and premise for further control and management. However, they were not fully accounted for in traditional methods. In this paper, with the consideration that network jam always stems from traffic jam formed within single road, attention was put on the traffic jam formation on signal controlled network comprised of basic road as in Figure 1 that is generally existed. After description of critical condition of jam formation for one-way link (Section 2.2), decomposition method is used to divide the road into two multilane segment (Section 2.3). Factors include insufficient split or excess demand (Section 3.1), endogenous flow (Section 3.2) and channelization spillback (Section 3.3) are discussed. The influences of road spillback on upstream links

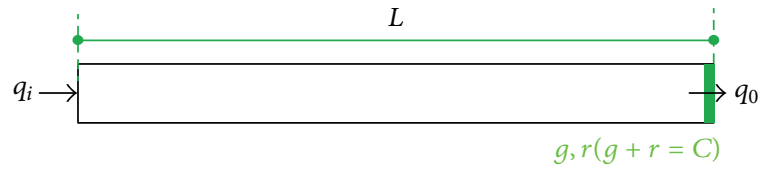

FIGURE 2: A Single one-way link.

are then analyzed (Section 4). We end with some highlights for further researches.

\section{Preliminary Results}

\subsection{Notations}

$q_{m}$ : optimal flow rate,

$k_{m}$ : optimal density,

$k_{j}$ : jam density,

$g, r, C, \lambda$ : green time, red time, cycle and split respectively,

$r_{v}$ : virtual red time,

$u_{0}, u_{1}, u_{2}$ : stopping wave, starting wave, and wave that emerges after $u_{0}$ and $u_{2}$ meet,

$l_{\max }$ : maximal queue length,

$q_{o l}, q_{o s}, q_{o r}$ : output flow rate of left-turn flow, through flow and right-turn flow. " $o$ " means output,

$g_{o l}, g_{o s}, g_{o r}$ : green time for left-turn flow, through flow and right-turn flow.

2.2. Wave Dynamics under Simple Condition. Consider a onelane road controlled by signal without endogenous flow as shown in Figure 2 which is the simplest condition in the road network (such as a road regulated by one-way traffic organization), assuming that fundamental diagram of traffic flow is parabolic function as shown in Figure 3. Upstream flow is $q$ (point A in Figure 2) and from parabolic function $k$ can be obtained (density of upstream flow is always smaller than optimum density under maximum flow; that is, point $\mathrm{A}$ should be on left side of the curve, otherwise stopping wave speed would be bigger than starting wave which denotes that queue would not disperse forever, which can be seen from Figure 3):

$$
k=\frac{k_{j}-\sqrt{k_{j}^{2}-4\left(k_{m}^{2} / q_{m}\right) q}}{2} .
$$

Formation and dispersion of a queue behind stop line at signal controlled intersection are shown in Figure 4(a). At first (the beginning of red time), stopping wave (red lines in Figure 4 which represent queue back) propagates upstream with velocity $u_{0}$ and a queue forms. When green time begins a starting wave (blue line in Figure 4) emerges and also propagates upstream with bigger speed (denoted as $u_{1}$ ). After $t^{\prime}$ starting wave catches up with stopping wave and queue has dispersed and a new wave (denoted by its speed $u_{2}$ ) forms. It takes $t^{\prime \prime}$ for wave $u_{2}$ to pass through stop line. If green time, $g$, 


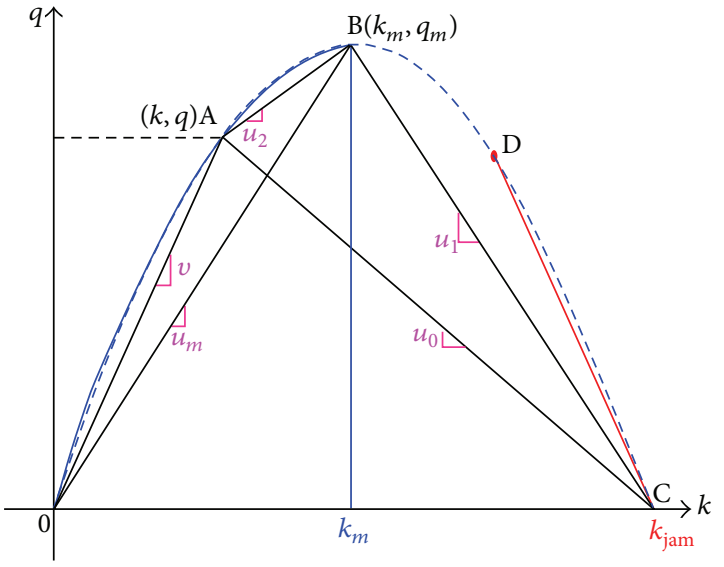

(a)

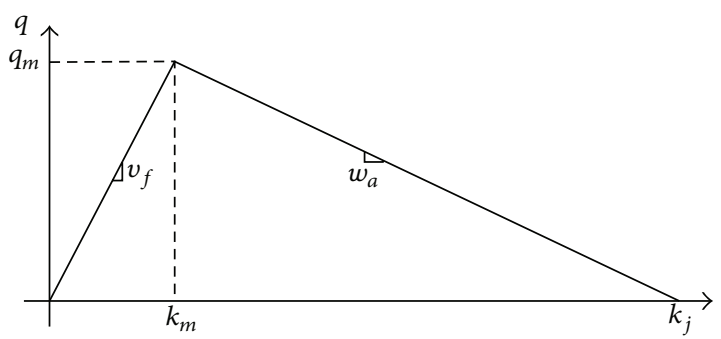

(b)

Figure 3: (a) Concave fundamental diagram; (b) triangular fundamental diagram.

is bigger than $t^{\prime}+t^{\prime \prime}$, then it can be concluded that traffic state would reproduce cycle by cycle. However, if $g$ is smaller than $t^{\prime}+t^{\prime \prime}$, the wave propagation profile is different (as shown in Figure 4(b)) cycle by cycle. At first, stopping wave will spread with speed $u_{1}$ which makes queue back of this cycle further from stop line than former cycle. As time elapses, queue back becomes further and further, so it is an unstable condition which represents oversaturated condition.

Based on the analysis above, some formulas can be got:

$$
\begin{gathered}
u_{1}=\frac{q_{m}}{k_{j}-k_{m}}, \quad u_{0}=\frac{q}{k_{j}-k}, \quad u_{2}=\frac{q_{m}-q}{k_{m}-k}, \\
u_{0}\left(r+t^{\prime}\right)=u_{1} t^{\prime} \Longrightarrow t^{\prime}=\frac{u_{0} r}{u_{1}-u_{0}}, \\
l_{\max }=u_{1} t^{\prime}=\frac{u_{1} u_{0} r}{u_{1}-u_{0}}, \\
t^{\prime \prime}=\frac{L_{\max }}{u_{2}} .
\end{gathered}
$$

When $g=t^{\prime}+t^{\prime \prime}$ holds (we can deduce that this equation determines a specific split $\lambda$ ) a stable state forms (if $\lambda$ decreases, it will result in oversaturation), that is,

$$
\begin{gathered}
l_{\max }=u_{1} t^{\prime}=\frac{u_{1} u_{0} r}{u_{1}-u_{0}}, \\
g=\frac{l_{\max }}{u_{1}}+\frac{l_{\max }}{u_{2}} .
\end{gathered}
$$

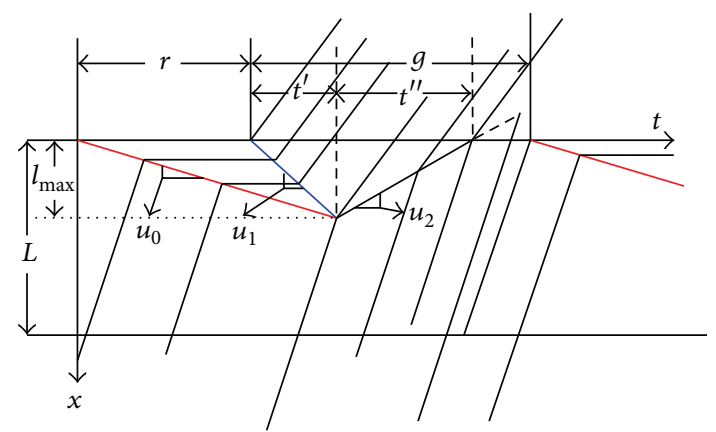

(a)

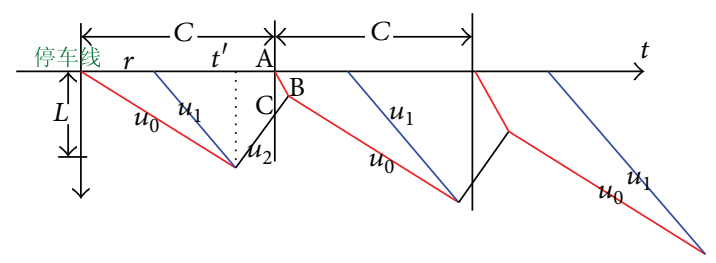

(b)

FIgURE 4: Traffic wave propagation.

The critical condition is defined such that the furthest queue back reaches road tail because larger $q$ or smaller split will result spillover of queue:

$$
\begin{gathered}
l_{\max }=u_{1} t^{\prime}=\frac{u_{1} u_{0} r}{u_{1}-u_{0}}, \\
g=\frac{l_{\max }}{u_{1}}+\frac{l_{\max }}{u_{2}}, \\
l_{\max }=L .
\end{gathered}
$$

Equations (4a), (4b), and (4c) establishe the relationship between link length $L$, cycle $C$, split $\lambda$, and flow $q$. Given $q$ split can be obtained as shown in embedded figure in Figure 5. When $q$ and $L$ are given, $\lambda$ and $g$ (also $C$ ) can be deduced as shown in Figure 5. It can be seen that links with different lengths require different control parameters and upstream inflow to maintain their normal operation. Much more green time is needed during higher demand under the same road length. This is why unchanged green time usually causes longer queue especially during peak hours. Spillover caused by smaller green time will be discussed later.

2.3. Decomposition of Complex Link Topology. The problems discussed above are mainly focusing on the ideal scenario of one-way links while the case in reality shows more topological complexity. We define the basic road shown in Figure 6(a) that always appears in urban road network. It is a road controlled by signal with a channelized section of length $l_{2}$. Overall upstream traffic demand, $q_{i}$ is assumed to be uniformly distributed between two lanes, that is, $q_{i 1}=q_{i 2}$. Proportion of left-turn flow, right-turn flow, and through flow is $p_{o l}, p_{o s}$, and $p_{o r}$ (and $p_{o r}=1-p_{o l}-p_{o s}$ ), respectively. The 


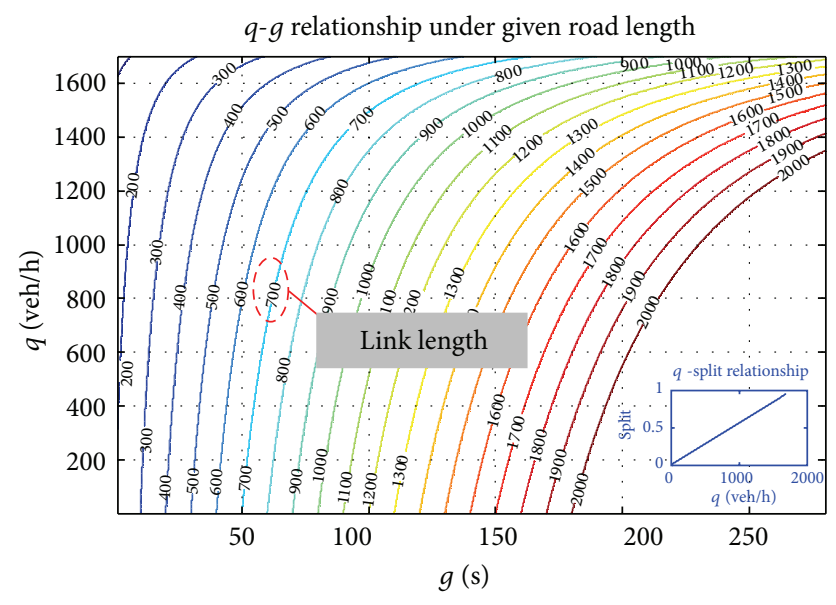

Figure 5: Critical $q-g$ relationship under different link length.

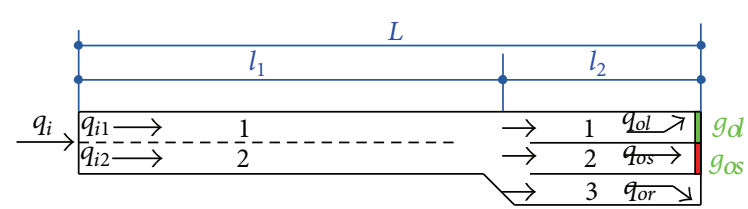

(a)

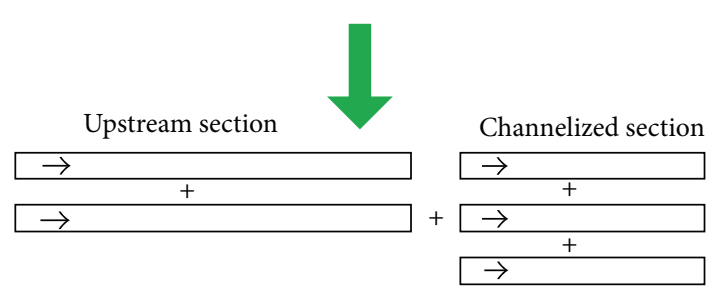

(b)

FIGURE 6: Sketch map of basic road and its decomposition.

same symbol expression method is for signal parameters, that is, $g_{o l}, g_{o s}, r_{o l}$, and $r_{o s}$, so we can get

$$
\begin{aligned}
q_{i}=q_{o l} & +q_{o s}+q_{o r}, \\
q_{o l} & =q_{i} p_{o l}, \\
q_{o s} & =q_{i} p_{o s}, \\
q_{o r} & =q_{i} p_{o r} .
\end{aligned}
$$

Decompose the basic road as shown in Figure 6(b). The basic road has been divided into two sections: upstream section and channelized section. Both are comprised of some single one-way links. Channelized sections are controlled by traffic signal (except right-turn section). The analysis hence is simplified that the method in Section 2 can be used in further analysis.

\section{Complex Circumstances}

During daily operation, circumstances often hold more complex nature. This section deals with this problem by analyzing

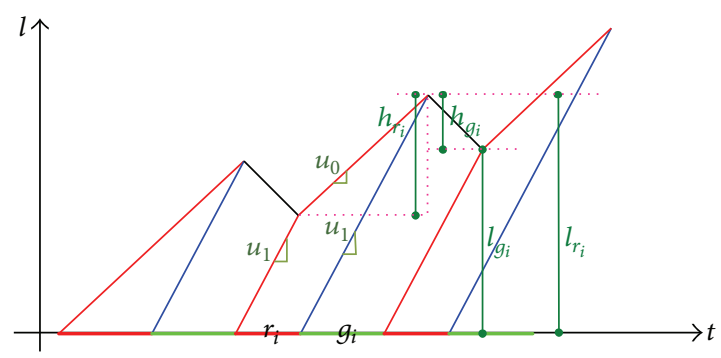

(a)

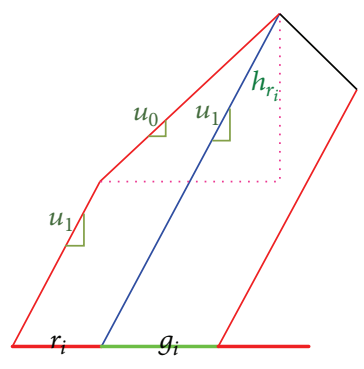

(b1)

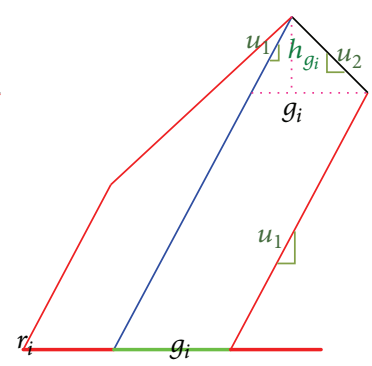

(b2)
FIGURE 7: Stopping wave and starting wave with insufficient split.

dynamic and insufficient split, endogenous flow of basic road, and the spillover of channelized section.

3.1. Dynamic and Insufficient Split. Usually oversaturation is developed gradually and queue back extends cycle by cycle, that is, domino phenomenon is the result of oversaturation extending in several cycles as shown in Figure 7(a). Each green signal tends to pull back queue back while each red signal prolongs queue back. Furthermore, the length pulled back or prolonged is proportional to time duration of traffic signal because in the figure slope of wave trajectory; that is, wave speed including starting wave and stopping wave is fixed.

Given green time $g_{i}$ of cycle $i$ (or red time $r_{i}$ ) queue length $h_{g_{i}}$ pulled back $\left(h_{r_{i}}\right.$ prolonged) can be computed from Figures $7(\mathrm{~b} 1)$ and 7(b2):

$$
\begin{aligned}
& h_{r_{i}}=\frac{r_{i} u_{0} u_{1}}{u_{1}-u_{0}}, \\
& h_{g_{i}}=\frac{g_{i} u_{2} u_{1}}{u_{1}+u_{2}} .
\end{aligned}
$$

After $i$ th red time $r_{i}\left(i\right.$ th green time $g_{i}$ ) location of queue back is

$$
\begin{gathered}
l_{r_{i}}=l_{0}+\sum_{i=1}\left(h_{r_{i}}-h_{g_{i}}\right)+h_{r_{i}}=l_{g_{i-1}}+h_{r_{i}}, \\
l_{g_{i}}=l_{0}+\sum_{i}\left(h_{r_{i}}-h_{g_{i}}\right),
\end{gathered}
$$

where $l_{0}$ denotes the length of initial oversaturated queue. It can also be calculated easily by assuming that the traffic is 


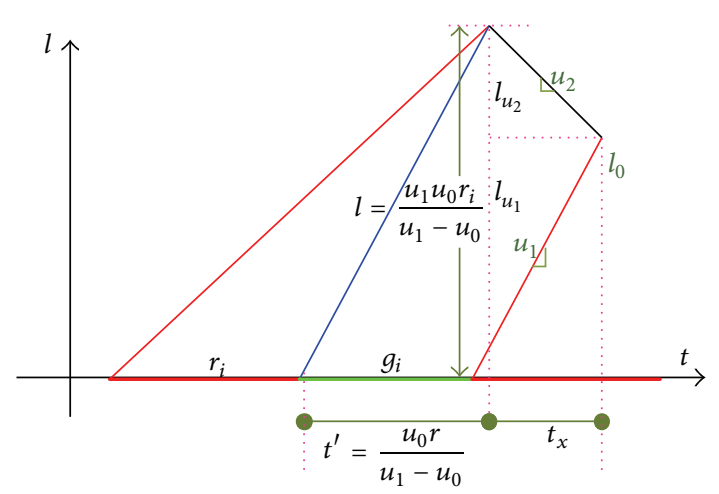

FIGURE 8: Initial oversaturated queue.

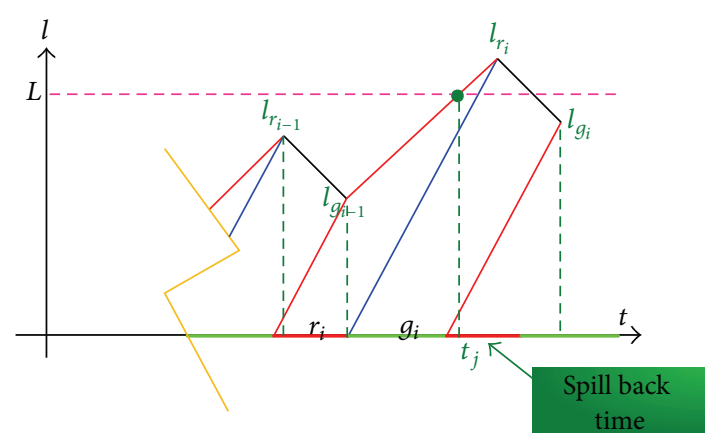

FIGURE 9: Spillover of road queue.

undersaturated at first, as shown in Figure 8. Symbols and derivations are as follows:

$$
\begin{gathered}
l_{u_{2}}+l_{u_{1}}=l, \\
u_{2} t_{x}+\left(t_{x}+t^{\prime}-g_{i}\right) u_{1}=l, \\
t_{x}=\frac{l-\left(t^{\prime}-g_{i}\right) u_{1}}{u_{1}+u_{2}}, \\
l_{0}=\left(t_{x}+t^{\prime}-g_{i}\right) u_{1}, \\
l_{0}=\left(\frac{l-\left(t^{\prime}-g_{i}\right) u_{1}}{u_{1}+u_{2}}+t^{\prime}-g_{i}\right) u_{1} .
\end{gathered}
$$

Replace $l_{0}$ in (7) queue back can be calculated at any time. Given changeable $r_{i}$ and $g_{i}$ and upstream traffic demand $q, h_{r_{i}}$ and $h_{g_{i}}$ hence each $l_{r_{i}}$ also can be determined. When queue back exceeds road length, that is, $l_{r_{i}}>L$ then spillover emerges $\left(t_{j}\right.$ in Figure 9 is the time when queue back exceeds road length).

However, spillover itself does not result domino phenomena because there may be no upstream traffic demand, this problem will be discussed in Section 4 .

In order to describe jam propagation upstream, the method of SVS (speed of virtual signal) is used. The principle is that there is a virtual signal located everywhere. When the jam has not reached a specific location, the virtual signal

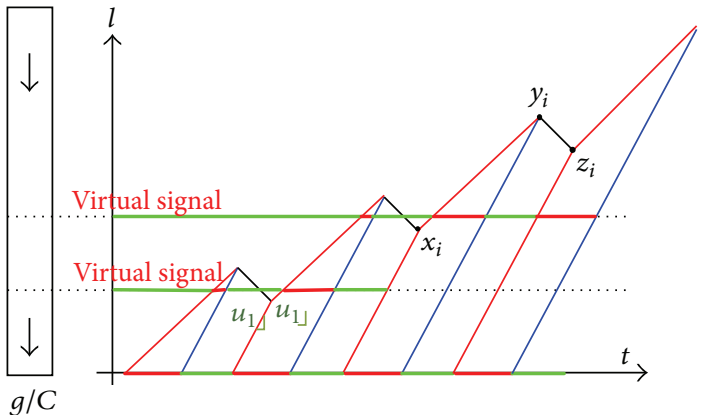

FIGURE 10: Method of virtual signal.

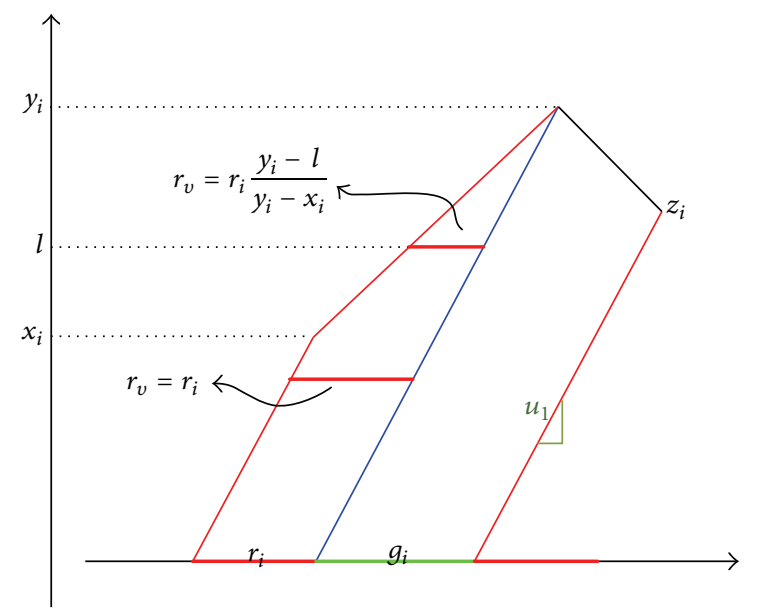

FIGURE 11: Virtual split calculation.

is always green. When the location is occupied by a queue (i.e., jam propagates to this position), the virtual signal shows red. When the queue is cleared, the virtual signal turns to green. From this description, we can see that the propagation of jam can be transformed to the propagation of virtual signal parameter. We use split to explore the propagation of jam. If we obtain locations of $x_{i}, y_{i}, z_{i}$ shown in Figure 10, then we can derive the split dynamics along time and space (see Figure 11). If demand exceeds supply, at the end of each cycle, the residual vehicle number is $C q-g q_{m}$ (the queue that remains after past cycles is not included in this formula), so three locations for each cycle can be derived:

$$
\begin{gathered}
x_{i}=x_{i-1}+\frac{C q-g q_{m}}{k_{j}}, \\
y_{i}=x_{i}+h_{r_{i}}, \\
z_{i}=y_{i}-h_{g_{i-1}} .
\end{gathered}
$$

After obtaining $x_{i}, y_{i}, z_{i}$ for every cycle, together with Figure 11, split everywhere for any cycle can be determined.

Figures 12 and 13 present a simple numerical example for propagation of split. From Figure 12, for a specified location, the gradual change of virtual signal cycle by cycle can be easily seen. Figure 13 gives the split on space-time (expressed as 


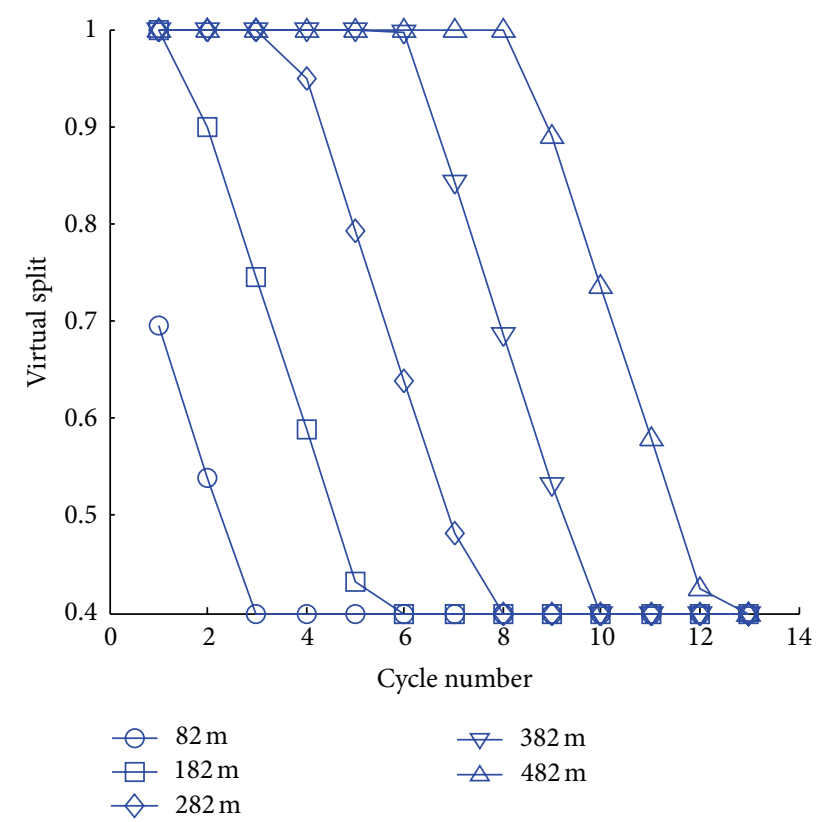

FIGURE 12: Virtual split propagation, $C=100 \mathrm{~s}, g=40 \mathrm{~s}, r=50 \mathrm{~s}$, $q=1000 \mathrm{ve} / \mathrm{h}, k_{j}=180 \mathrm{veh} / \mathrm{km}, q_{m}=1800 \mathrm{veh} / \mathrm{h}$.

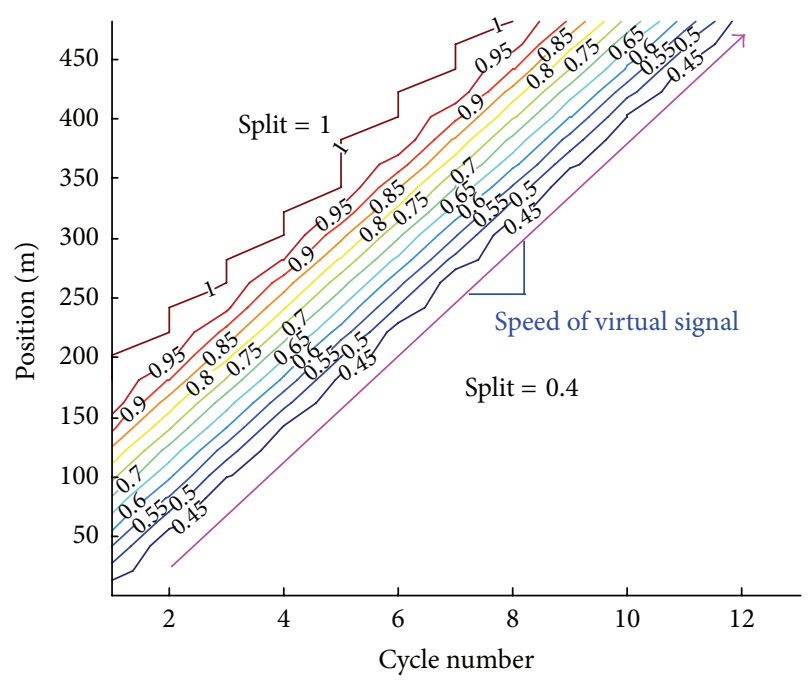

FIGURE 13: Contour of split on space-time diagram and parameters are the same as in Figure 12.

cycle number) diagram. The slope can be seen as the speed of jam propagation.

In order to develop a measure of this speed, an index which incorporates stopping wave and starting wave is proposed later on. For a cycle of jam propagation, the increment of queue back can be got:

$$
\Delta l_{i}=h_{r_{i}}-h_{g_{i-1}} .
$$

If the control parameters are constant, then we define speed of virtual signal (SVS) to express the severity of jam,

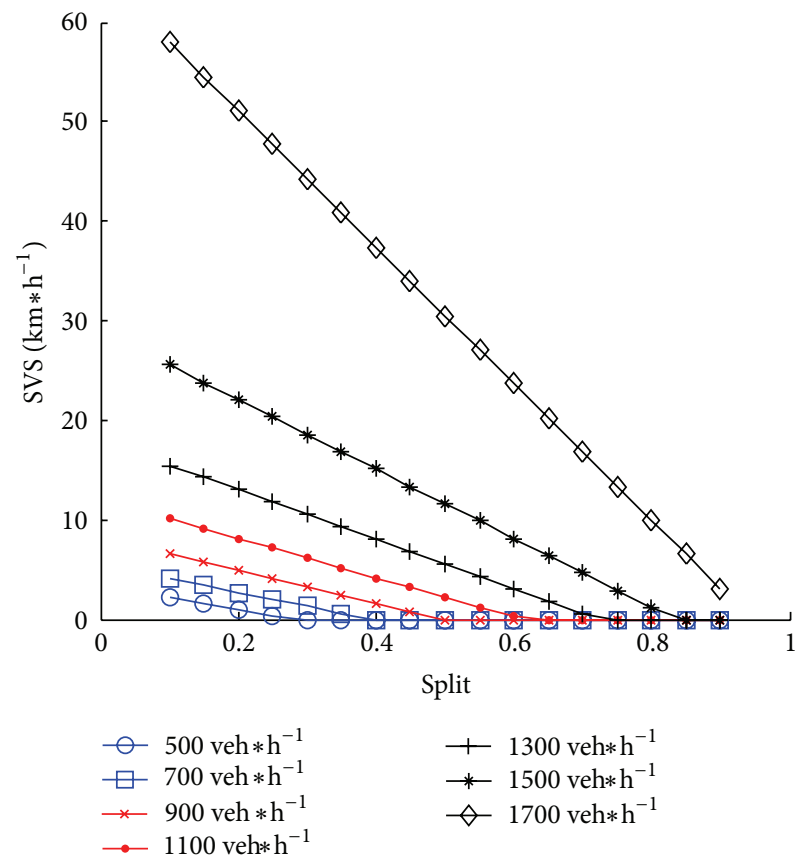

FIGURE 14: SVS versus split; $k_{j}=180 \mathrm{veh} / \mathrm{km}, q_{m}=1800 \mathrm{veh} / \mathrm{h}$.

that is, the propagation of congestion. During a cycle, queue back propagates $\Delta l_{i}$, and the average speed is then

$$
\begin{gathered}
\text { SVS }=\frac{\Delta l_{i}}{C}=\frac{r_{i} u_{0} u_{1} /\left(u_{1}-u_{0}\right)-g_{i} u_{2} u_{1} /\left(u_{1}+u_{2}\right)}{C} \\
=\frac{(1-\lambda) u_{0} u_{1}}{u_{1}-u_{0}}-\frac{\lambda u_{2} u_{1}}{u_{1}+u_{2}}, \\
\text { SVS }=\frac{u_{0} u_{1}}{u_{1}-u_{0}}-\lambda\left[\frac{u_{2} u_{1}}{u_{1}+u_{2}}+\frac{u_{0} u_{1}}{u_{1}-u_{0}}\right]=f(q, \lambda) .
\end{gathered}
$$

From the expression we can see that SVS is in linear relationship with split.

Figures 14 and 15 present the relationship between SVS and split, arriving flow rate, respectively. From the figure we can see that SVS is very sensitive to traffic state under saturation condition. The results above are based on parabolic fundamental, which is symmetrical about optimal flow. Figures 16 and 17 plot the SVS versus split and arriving flow under triangular fundamental diagram like Figure 3(b). The parameters are given beneath the figure.

Similar trend is also found for triangular fundamental diagram. However, it is evident from both figures that change of SVS is somewhat moderate compared with parabolic diagram. This phenomena stem from the different nature embedded in these two fundamental diagrams: wave speed for triangular diagram is constant when the density is below $k_{m}$, but for parabolic fundamental diagram, wave speed (tangent line of specific point) is changing simultaneously with traffic demand.

Equation (12) assumes that the arriving flow is constant; however, it is not the case. But we can generalize it to dynamic 


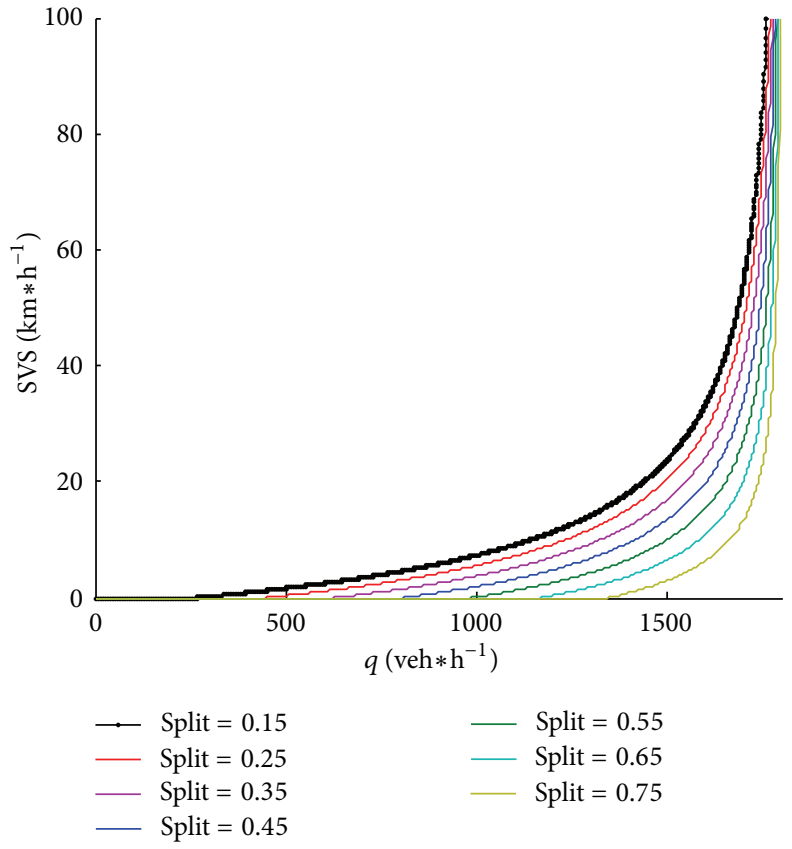

FIGURE 15: SVS versus arriving flow rate; $k_{j}=180 \mathrm{veh} / \mathrm{km}, q_{m}=$ $1800 \mathrm{veh} / \mathrm{h}$.

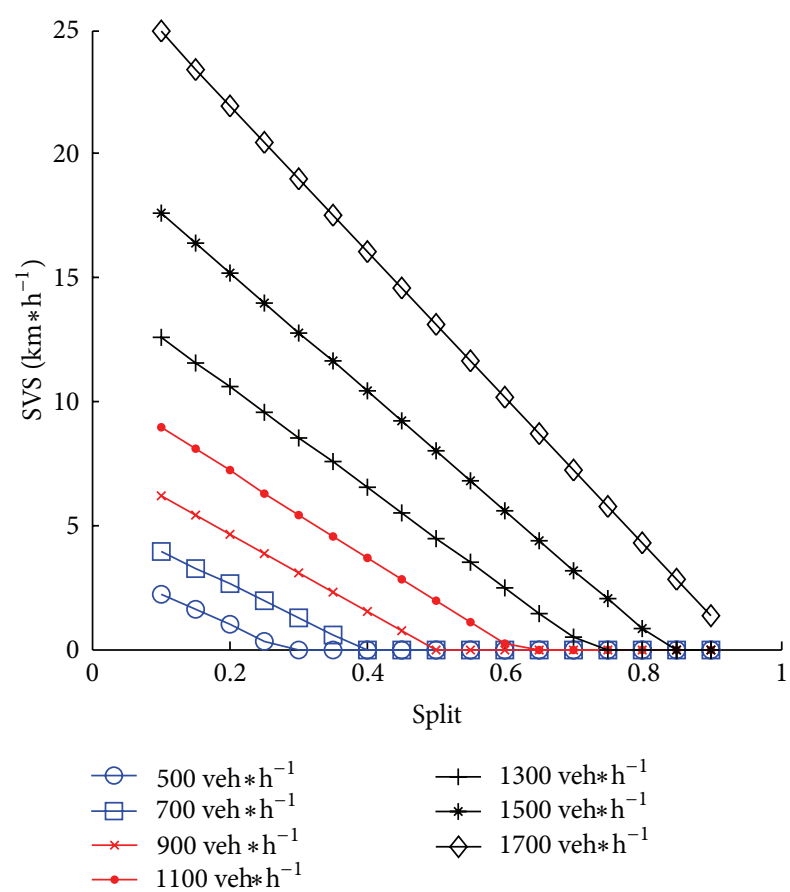

FIGURE 16: SVS versus split with triangular fundamental diagram; $k_{j}=180 \mathrm{veh} / \mathrm{km}, q_{m}=1800 \mathrm{veh} / \mathrm{h}, v_{f}=50 \mathrm{~km} / \mathrm{h}$, and $w_{a}=$ $12.5 \mathrm{~km} / \mathrm{h}$.

condition where within one cycle the arriving is stable but different from cycle to cycle, that is,

$$
\mathrm{SVS}=\frac{u_{0}(i) u_{1}}{u_{1}-u_{0}(i)}-\lambda\left[\frac{u_{2}(i) u_{1}}{u_{1}+u_{2}(i)}+\frac{u_{0}(i) u_{1}}{u_{1}-u_{0}(i)}\right],
$$

where $u_{0}(i)$ is stop wave speed at cycle $i$.

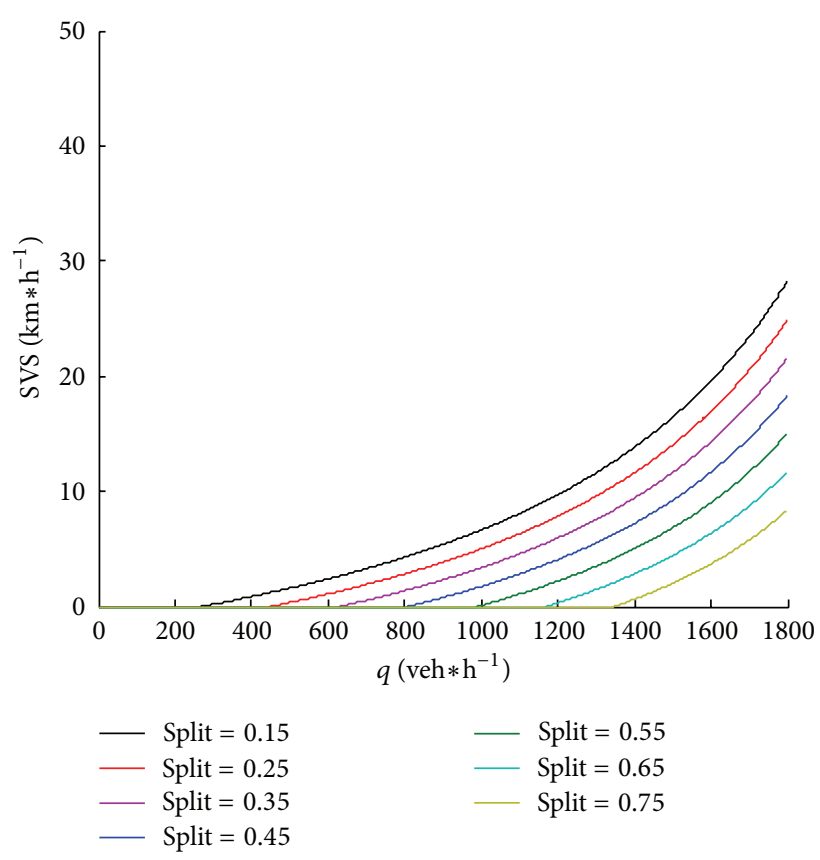

FIGURE 17: SVS versus arriving flow rate with triangular fundamental diagram; $k_{j}=180 \mathrm{veh} / \mathrm{km}, q_{m}=1800 \mathrm{veh} / \mathrm{h}, v_{f}=50 \mathrm{~km} / \mathrm{h}$, and $w_{a}=12.5 \mathrm{~km} / \mathrm{h}$.

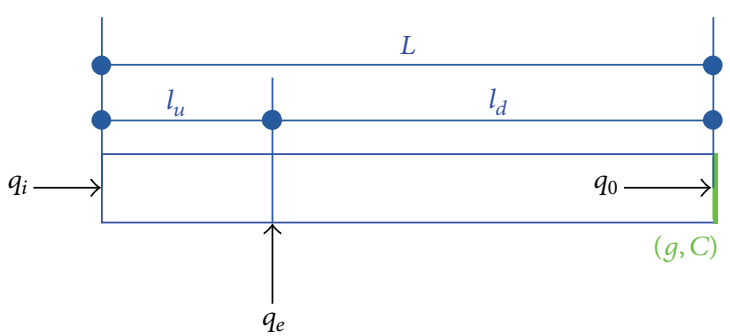

Figure 18: Single road with endogenous traffic flow.

3.2. Influence of Endogenous Flow. Traditionally it is assumed that flow is generated at nodes (i.e., intersections within the network or originations of a road network) as we did in network traffic analysis [18]. However, intersections inside road network actually do not generate flow (of course, from the viewpoint of downstream intersection, flow is "produced" at upstream intersection). In fact, most traffic stems from entrance within road (e.g., shopping malls, schools, and official departments about the road). It is necessary to take endogenous traffic flow into account. Due to complexity in analyzing multilane road, we only deal with one-way road with entrance inside which the flow is denoted as $q_{e}$ (shown in Figure 18). The basic assumption is major road priority; that is, vehicles in major road have priority to cross.

When a formed queue back has not reached entrance location, the upstream flow is $q_{i}+q_{e}$; when queue length exceeds $l_{d}$ (distance between stop line and endogenous flow entrance location) upstream flow becomes $q_{i}$, so the stopping wave and starting wave propagate differently from that without endogenous flow (Figure 4(a)). Given $q_{i}$ and 


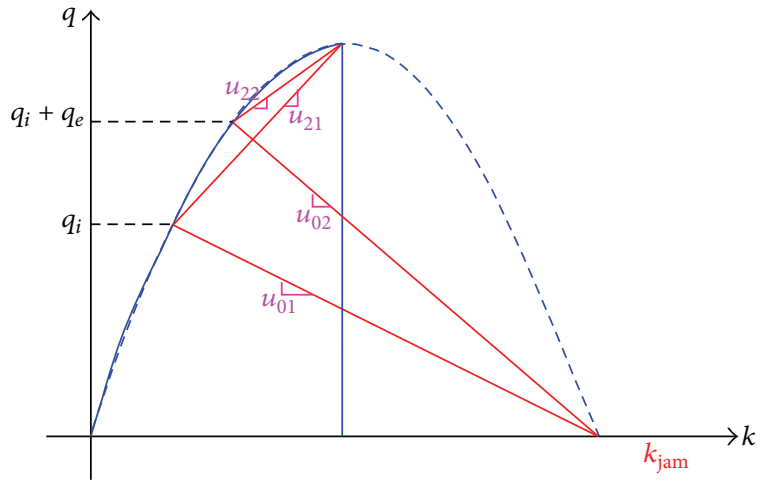

FIgURE 19: Fundamental diagram.

$q_{e}$ stopping wave profile and starting wave profile can be obtained using the same method. Denote stopping wave as $u_{01}$ (wave when queue length has exceeded $l_{d}$ ), $u_{02}$ (wave when queue length has not exceeded $l_{d}$ ) and $u_{21}, u_{22}$ as the wave produced after $u_{02}$ and $u_{1}$ meet as shown in Figure 20.

From Figure 19 formulas below can be got:

$$
\begin{gathered}
u_{01}=\frac{\left(q_{i}+q_{e}\right)}{k_{j}-k}, \quad u_{02}=\frac{q_{i}}{k_{j}-k}, \\
u_{21}=\frac{q_{m}-q_{i}}{k_{m}-k}, \quad u_{22}=\frac{q_{m}-\left(q_{i}+q_{e}\right)}{k_{m}-k} .
\end{gathered}
$$

For case I, critical condition can be formulated just as in (3a) and (3b):

$$
\begin{gathered}
l_{\max }=u_{1} t^{\prime}=\frac{u_{1} u_{02} r}{u_{1}-u_{02}}, \\
g=\frac{l_{\max }}{u_{1}}+\frac{l_{\max }}{u_{22}} .
\end{gathered}
$$

For cases II and III, critical conditions as follows:

$$
\begin{gathered}
r_{v}=r-l_{d}\left(\frac{u_{1}-u_{02}}{u_{02} u_{1}}\right), \\
l_{\max }=l_{d}+\frac{u_{1} u_{01} r_{v}}{u_{1}-u_{01}}, \\
g=\frac{u_{1} u_{01} r_{v} /\left(u_{1}-u_{01}\right)}{u_{21}}+\frac{l_{d}}{u_{22}} .
\end{gathered}
$$

The virtual red time in Figure 20 and (16a), (16b), and (16c) is obtained from geometric relationship. Figure 21 gives the relationship between critical split and different entrance locations from (15a), (15b), (16a), (16b), and (16c). We can see that upstream location gets more influence in that once queue propagates exceed entrance endogenous flow cannot enter. Moreover, propagation of jam (i.e., SVS under this condition) also holds such trend because of the same tendency of propagation speed of stopping and starting wave.

3.3. Channelized Section Spillover. Most researches do not take spillover of channelized section into account. But at peak hours due to length constraint the channelized section often cannot accommodate excessive vehicles which make spillover of channelized section inevitable. Following Wright and Roberg [1], we assume that traffic flow running on the upstream section was mixed uniformly (i.e., velocity, density, and flow are uniformly distributed in the section) and lane changing are executed instantly at interface between channelized section and upstream section. This is acceptable from the viewpoint of system description although some details we do not concern with are lost. Road layout is as that in Figure 6. From fundamental traffic theory we can easily get flow-density relationship as shown in Figure 22. Parabolic curve beneath is flow-density relationship of leftturn channelized section (right-turn or through channelized section is the same) and the upper curve is that of upstream section.

When there exists channelized section spillover the influence can be considered as "virtual red time," principle of which is described in Figure 23: once there is spillover a queue will propagate upstream and block upstream section result of which is the same as signal control. A virtual signal is set at the interface between two sections as shown in Figure 23. Virtual red time equals to time difference between stopping wave and starting wave spreading over the interface. Now the analysis of spillover with consideration of channelized section can be taken by two steps:

(I) analyze supply-demand of channelized section to obtain block time (note that queues of two channelized sections, that is, through section and leftturn section may be overflowed at the same time or may be not, so block time of these spillovers may be separated, overlapped, or overlapped partly);

(II) after virtual time (interface block time) is obtained, analyze supply-demand of upstream section. If upstream section queue back exceeds road length, then upstream intersection will be blocked.

Due to the fact that both channelized sections may block the interface we first deal with only one section spillover and then two.

3.3.1. One Section Spillover. Since results of spillover of the two channelized section are the same, here we simply assume that only left-turn section spillover takes place. When $p_{o l}=$ $p_{o s}$, that is, left-turn traffic flow $q_{o l}$ equals through flow $q_{o s}$. It can be deduced from Figure 22 that starting wave and stopping wave keep its speed after spread over interface. It is condition A in Figure 24. This condition degrades to one-way link spillover which has been discussed in Section 2.

When $p_{o l}>p_{o s}$ (i.e., $q_{o l}>q_{o s}$ ), upstream section holds more flow averagely than channelized section. The wave propagation profile should be the pattern as B in Figure 24. Its stopping wave speed $u_{0 a}$ is bigger than $u_{0}$ and $u_{2 a}$ is smaller than $u_{2}$. When green time $g_{o l}$ extends over $Z$, the queue will be cleared completely. From the figure critical conditions for domino formation are deduced:

$$
\left(\frac{l_{2}}{u_{0}}+\frac{l_{1}}{u_{0 a}}\right)+\left(\frac{l_{1}}{u_{2 a}}+\frac{l_{2}}{u_{2}}\right)=r_{o l}+g_{o l} \text {. }
$$




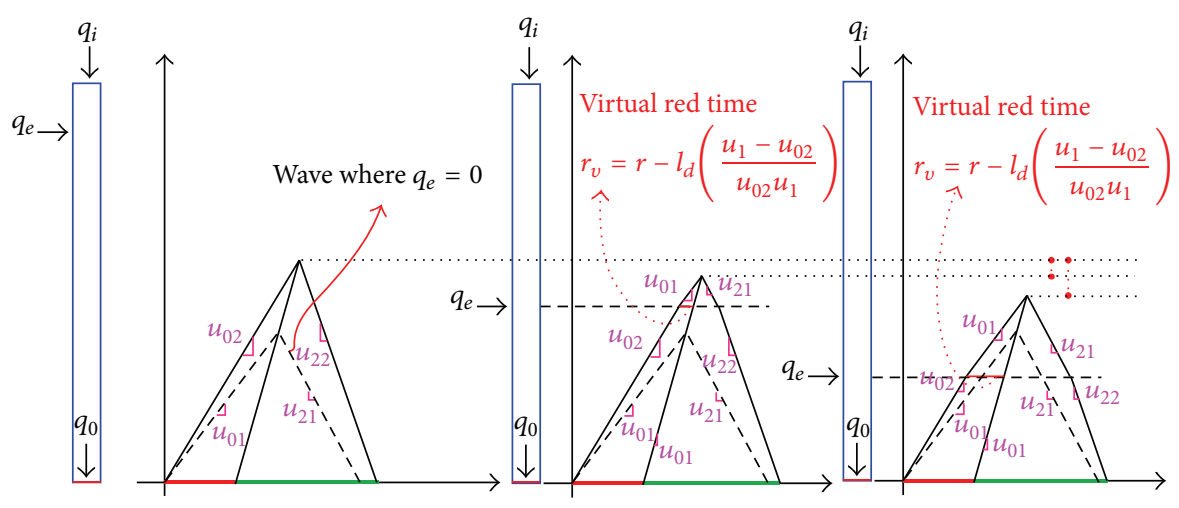

(I)

(II)

(III)

FIGURE 20: Stopping wave and starting wave with endogenous flow.

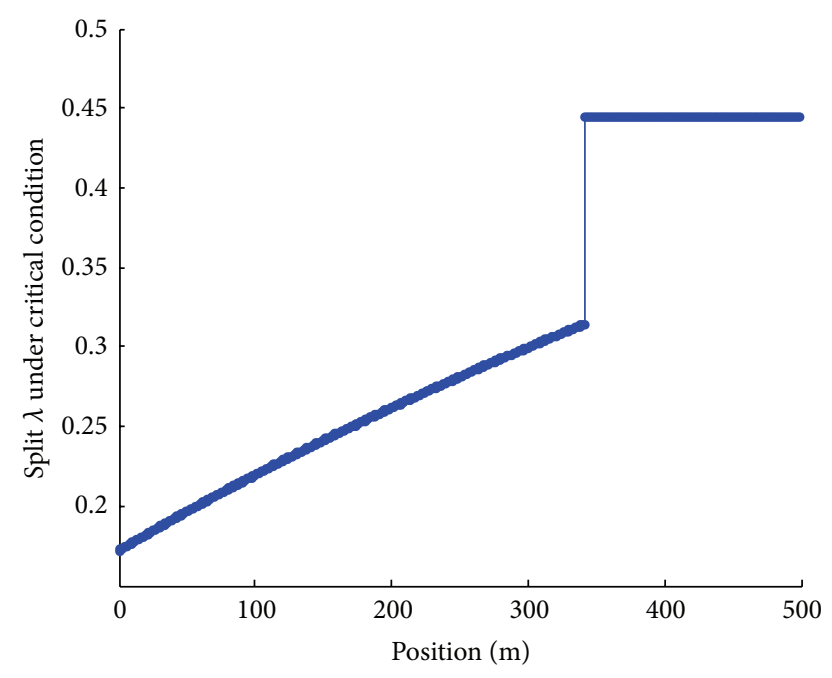

FIGURE 21: Critical split for jam formation versus different entrance position $\left(l_{d}\right), q_{i}=500 \mathrm{veh} / \mathrm{h}, q_{e}=300 \mathrm{veh} / \mathrm{h}$.

When $p_{o l}<p_{o s}$ (i.e., $q_{o l}<q_{o s}$ ), upstream section holds less flow averagely than channelized section, so the wave propagation profile should be the pattern as $C$ in Figure 24. Its stopping wave speed $u_{0 c}$ is smaller than $u_{0}$ and $u_{2 c}$ is greater than $u_{2}$. When green time $g_{o l}$ extends over $X$ then the queue will be cleared completely. Critical conditions for domino formation are deduced:

$$
\left(\frac{l_{2}}{u_{0}}+\frac{l_{1}}{u_{0 c}}\right)+\left(\frac{l_{1}}{u_{2 c}}+\frac{l_{2}}{u_{2}}\right)=r_{o l}+g_{o l} .
$$

From geometry and Figure 24, we can deduce virtual red time for interface when critical condition (i.e., (17) and (18)) is not reached:

$$
r_{v}=r_{o l}+\frac{l_{2}}{u_{1}}-\frac{l_{2}}{u_{0}}=r_{o l}-l_{2}\left(\frac{u_{1}-u_{0}}{u_{0} u_{1}}\right),
$$

where $r_{v}$ is virtual red for interface. From it split can also be determined: $\lambda_{v}=\left(C-r_{v}\right) / C$. Since $\left(u_{1}-u_{0}\right) / u_{0} u_{1}>0$, from

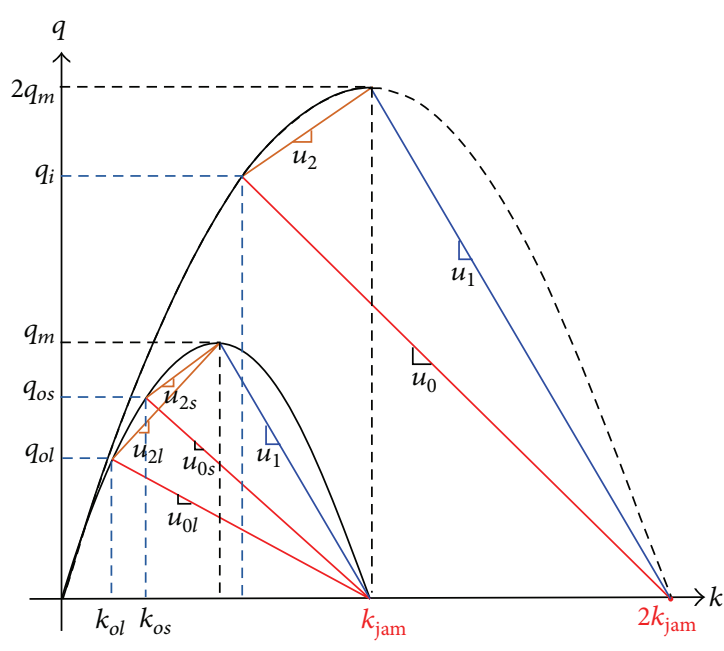

FIGURE 22: $q-k$ relationship of two sections.

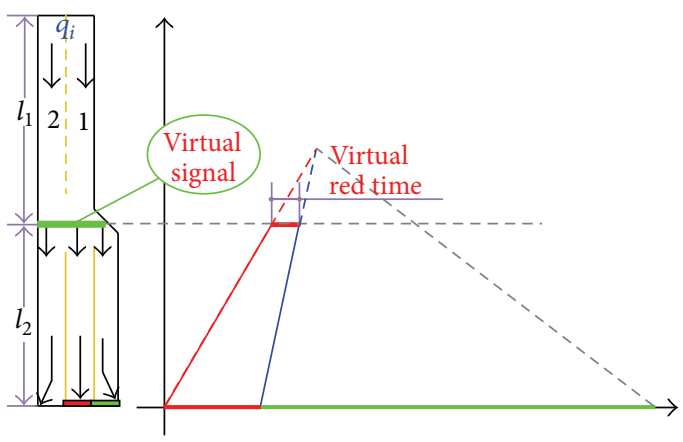

FIGURE 23: Virtual signal and virtual red time.

(19) we can see that virtual red time increases against channelization section length, and opposite relationship holds for split and channelization section length.

Now critical condition of (4a), (4b), and (4c) can be used to determine supply-demand of upstream section. Note that there is no channelization spill back for through traffic flow. Since virtual split of interface is bigger than $\left(C-r_{o l}\right) / C$ (from 


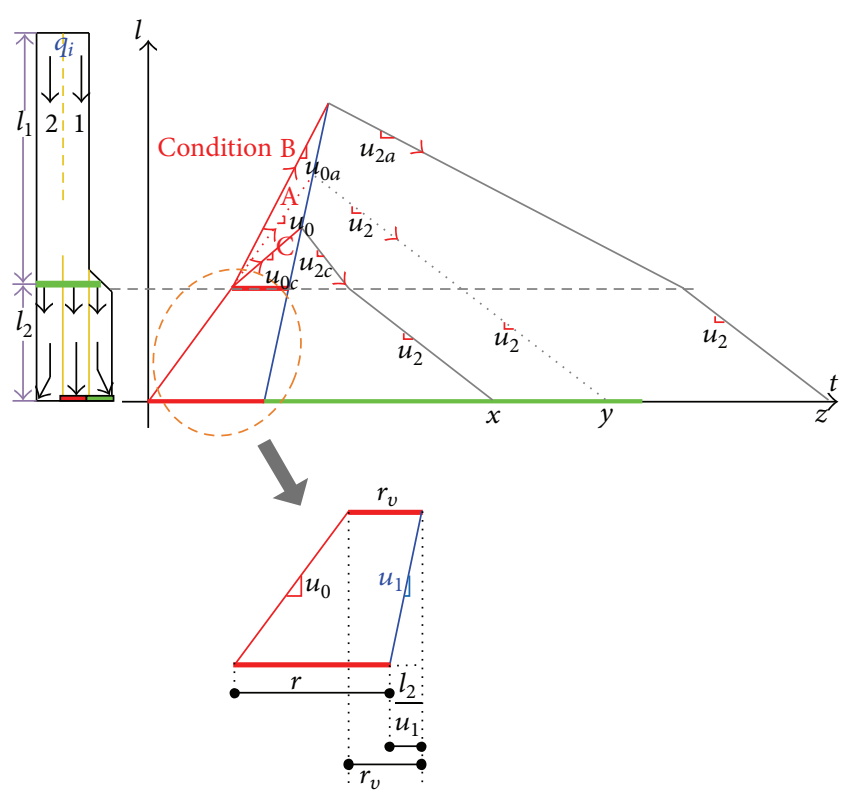

FIGURE 24: Wave profile when channelized section spillover exists.

(19) relationship that $r_{v}<r_{o l}$ is evident and also $\lambda_{v}<\lambda_{o l}$ ), for condition A and Cin Figure 24, if critical condition of (18) has not reached, demand of upstream section is always satisfied because

$$
\frac{q_{o l}+q_{o s}}{\lambda_{v} q_{m}+\lambda_{v} q_{m}}<\frac{2 q_{o l}}{2 \lambda_{v} q_{m}}=\frac{q_{o l}}{\lambda_{v} q_{m}}<\frac{q_{o l}}{\lambda_{o l} q_{m}} .
$$

For condition B, if the critical relationship of (4a), (4b), and $(4 \mathrm{c})$ is not hold, traffic jam will form and propagate upstream.

If channelization spillover of only one direction happens, capacity of the other two directions may be wasted, which imply the importance of well-designed phase sequences. From (4a), (4b), and (4c), it implies that the equation below should hold to prevent wasted capacity:

$$
\begin{gathered}
l_{\max }=u_{1} t^{\prime}=\frac{u_{1} u_{0} r_{o l}}{u_{1}-u_{0}}, \\
g_{o l}>\frac{l_{\max }}{u_{1}}+\frac{l_{\max }}{u_{2}}, \\
l_{\max }<l_{2} .
\end{gathered}
$$

Equations (21a) and (21b) assure that the demand can be satisfied and (21c) guarantees queue back would not reach interface such that block cannot happen.

3.3.2. Two Section Spillovers. Sometimes during peak hour left-turn demand and through demand may both exceed respective capacity which results in spillover of both sections. In such case the problem may be complicated but can be explained conveniently. Spillover of two sections is just as we set two signals in the interface at the same time. When both virtual signals show green then overall virtual signal is

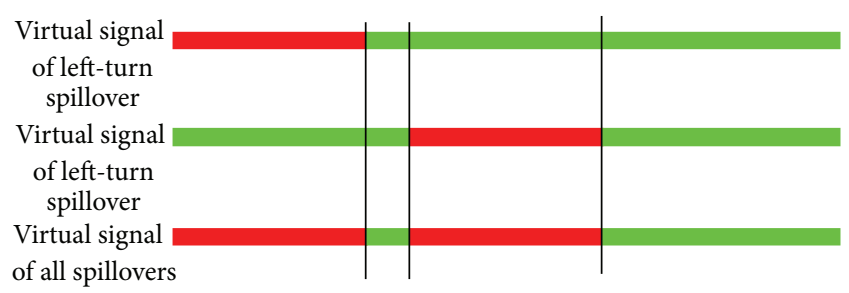

FIGURE 25: Virtual signal of both spillover.
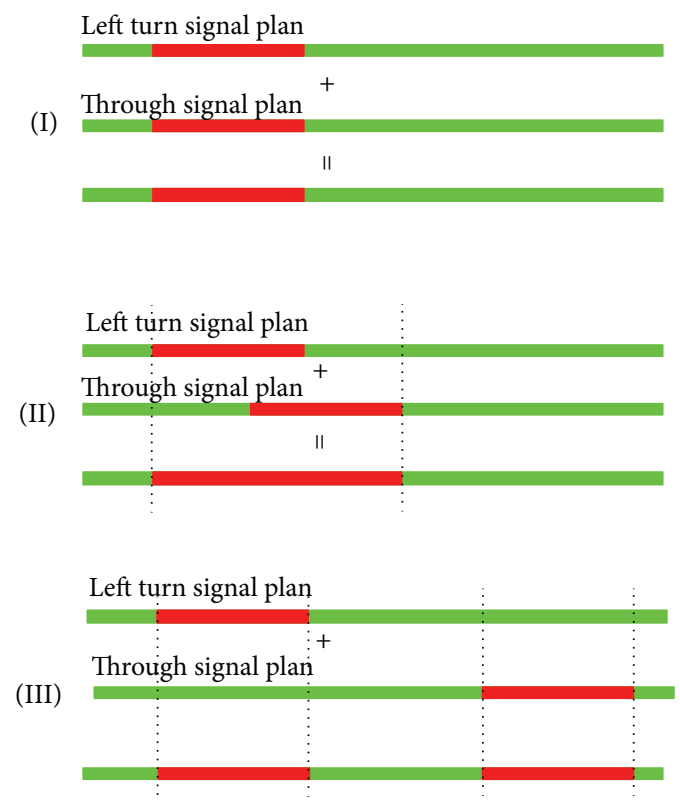

FIGURE 26: Virtual signal for three cases.

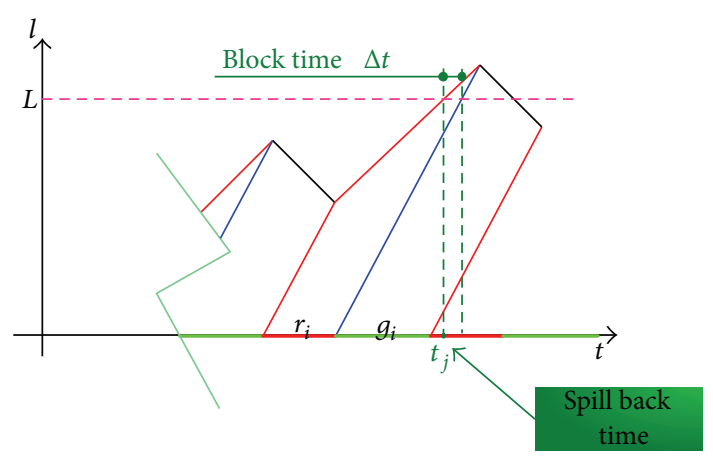

FIGURE 27: Spillover time and block time.

green otherwise the overall signal is red. One case is shown in Figure 25.

According to the signal assignment of left-turn flow and through flow, there will be three cases as shown in Figure 26. Apparently for the same split, different "offset" will produce different spillover virtual signal which implies the importance of phase sequence under spillover condition. We can see that during congestion hours (where channelized section spill back take place); it would be better to put through flow and left-turn flow in the same phase. 


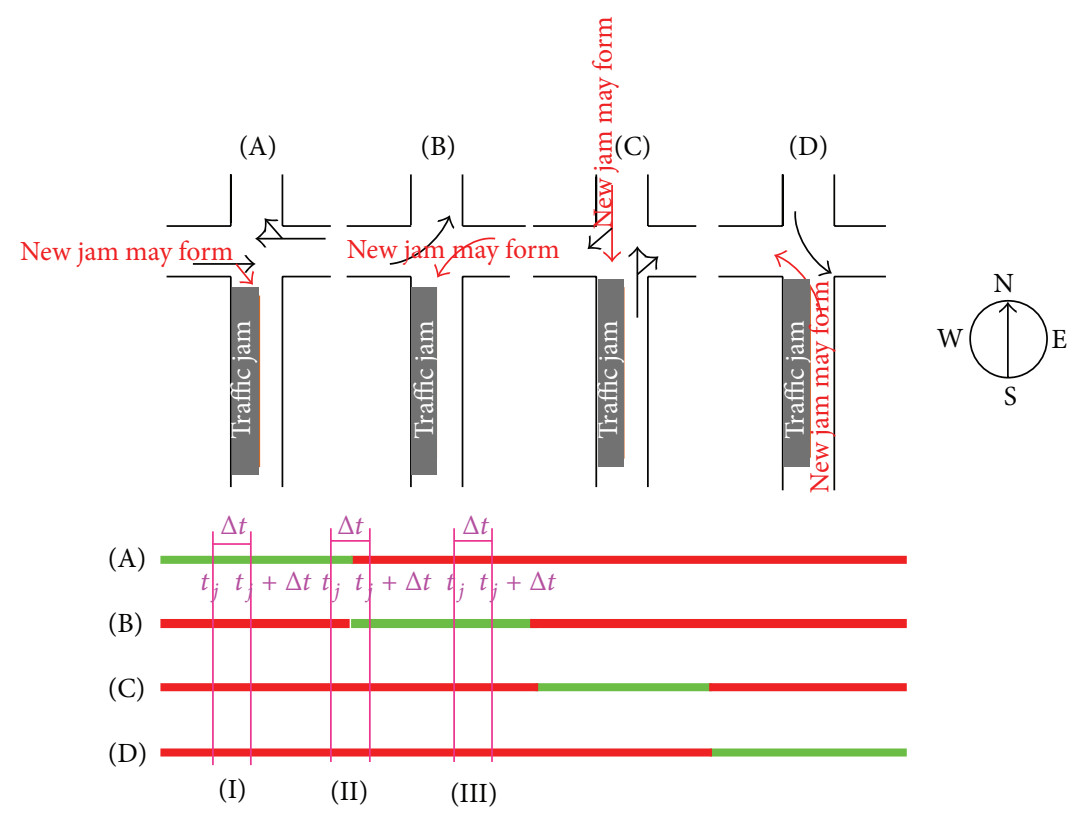

FIGURE 28: Block of upstream intersection.

\section{Impact of Spillover on Upstream Intersection}

Spillover is the precondition of congestion propagation. However, spillover itself is not enough for domino phenomena formation because if there is not enough upstream demand congestion would not spread. Thus the sufficient and necessary condition for domino phenomena is first, spillover of downstream road and second, there is continuous demand upstream.

When a queue forms and spill back and blocks upstream intersection, there are basically two events to be analyzed: first, how long it will block upstream intersection and second, its influence on upstream flows. When queue back reaches upstream intersection at time, say, $t_{j}$ (as shown in Figure 27) then it blocks the intersection till the starting wave catches up with it.

We denote that the time duration of the intersection is blocked (i.e., blocked time) as $\Delta t$, then from the viewpoint of upstream signal phases there are three types (using a typical four-phase intersection shown in Figure 28 as an example) of block. Take phase A to describe as following.

(I) Block fully happens at green time. Then the phase will lose $\Delta t$ effective green time which will decrease capacity respectively.

(II) Block fully happens at red time. Since no matter whether the intersection is blocked, this movement always has not got RoW (Right of Way), so this type of block holds no impact.

(III) Block happens partially at green time and partially at red time (let us neglect amber time because it will not harm the conclusion). This block will encroach some green time which will reduce some capacity.

Due to reduced capacity caused by downstream block, specific phase upstream will be under oversaturation temporarily and long queue forms which may be block upstream intersection of this crossroad.

The block influences upstream link flow differently. In Figure 28 the most possible flow blocked by downstream jam is highlighted in red line for each phase. Green time for these flows then may be wasted hence new jam may form at upstream links. This can be seen as domino phenomena of traffic jam in the whole network.

From the description above we can see that badly designed signal may contribute to network jam propagation, which suggests the importance of network signal coordination during peak hours.

\section{Conclusion}

Due to the complexity of urban traffic jam, many researches were taken under simplified assumption or used simulated methods. In this paper, urban traffic jam formation is studied. From the research we can see that many factors (such as road geometry, flow structure, and signal settings) contribute to the traffic jam formation and propagation which should be took into account in the control of jam during peak hours.

However, limited by the macroscopic method, we cannot look into deeply mutual influence of different flow, (leftturn flow, right-turn flow, and through flow) which may contribute to the formation and propagation of traffic jam. This may be solved by using microscopic analysis methods. Furthermore, formation of congestion is only prelude of 
large scale urban traffic jam. Propagation of urban traffic jam (denominated as "domino phenomena" here) itself is influenced by many factors. Some further researches could be highlighted.

(1) The calibration of fundamental diagram: the relationship between flow, density, and speed is a basic problem of traffic engineering field and exerts great influence on jam propagation (severity and propagation speed). A thorough examination of the influence is needed.

(2) The asssumption of uniformly mixed flow at upstream section shoule be revised. In reality, the distribution may be not averagely. Whether this feature prompts the propagation $\mathrm{f}$ traffic jam can be included in our model with a coefficient that needs to be explored closely.

(3) Isolated intersection signal design at peak hours. From this research we can see that traditional control principle of minimizing delay may be ineffective in dealing with peak hour flow due to the spillover of long queue. Till now the control strategy that takes this condition into account is absent.

\section{Acknowledgments}

This work was supported by the State Key Development Program for Basic Research of China (Grant no. 2012CB725402); the National Science Foundation for Postdoctoral Scientists of China (Grant no. 2012M521175); Excellent Postdoctoral Science Foundation of Zhejiang Province (Bsh1202056).

\section{References}

[1] C. Wright and P. Roberg, "The conceptual structure of traffic jams," Transport Policy, vol. 5, no. 1, pp. 23-35, 1998.

[2] M. Schreckenberg, L. Neubert, and J. Wahle, "Simulation of traffic in large road networks," Future Generation Computer Systems, vol. 17, no. 5, pp. 649-657, 2001.

[3] M. Treiber, A. Hennecke, and D. Helbing, "Congested traffic states in empirical observations and microscopic simulations," Physical Review E - Statistical Physics, Plasmas, Fluids, and Related Interdisciplinary Topics, vol. 62, no. 2 B, pp. 1805-1824, 2000.

[4] N. J. Linesch and R. M. D'Souza, "Periodic states, local effects and coexistence in the BML traffic jam model," Physica A: Statistical Mechanics and its Applications, vol. 387, no. 24, pp. 6170-6176, 2008.

[5] T. Nagatani, "The physics of traffic jams," Reports on Progress in Physics, vol. 65, no. 9, pp. 1331-1386, 2002.

[6] C. F. Daganzo, "The cell transmission model: A dynamic representation of highway traffic consistent with the hydrodynamic theory," Transportation Research Part B, vol. 28, no. 4, pp. 269287, 1994.

[7] C. F. Daganzo, "The cell transmission model, part II: Network traffic," Transportation Research Part B, vol. 29, no. 2, pp. 79-93, 1995.

[8] M. J. Lighthill and G. B. Whitham, "On kinematic waves. II. A theory of traffic flow on long crowded roads," Proceedings of the Royal Society. London. Series A. Mathematical, Physical and Engineering Sciences, vol. 229, pp. 317-345, 1955.
[9] P. I. Richards, "Shock waves on the highway," Operations Research, vol. 4, pp. 42-51, 1956.

[10] J. Long, Z. Gao, X. Zhao, A. Lian, and P. Orenstein, "Urban traffic jam simulation based on the cell transmission model," Networks and Spatial Economics, vol. 11, no. 1, pp. 43-64, 2011.

[11] P. Roberg and C. R. Abbess, "Diagnosis and treatment of congestion in central urban areas," European Journal of Operational Research, vol. 104, no. 1, pp. 218-230, 1998.

[12] P. G. Michalopoulos, D. E. Beskos, and J.-K. Lin, "Analysis of interrupted traffic flow by finite difference methods," Transportation Research. Part B. Methodological. An International Journal, vol. 18, no. 4-5, pp. 409-421, 1984.

[13] A. Skabardonis and N. Geroliminis, "Real-time estimation of travel times along signalized arterials," in Proceedings of the 16th International Symposium on Transportation and Traffic Theory, 2005.

[14] A. Skabardonis and N. Geroliminis, "Real-time monitoring and control on signalized arterials," Journal of Intelligent Transportation Systems: Technology, Planning, and Operations, vol. 12, no. 2, pp. 64-74, 2008.

[15] C. F. Daganzo, "Urban gridlock: Macroscopic modeling and mitigation approaches," Transportation Research Part B: Methodological, vol. 41, no. 1, pp. 49-62, 2007.

[16] X. Wu, H. X. Liu, and D. Gettman, "Identification of oversaturated intersections using high-resolution traffic signal data," Transportation Research Part C: Emerging Technologies, vol. 18, no. 4, pp. 626-638, 2010.

[17] N. Geroliminis and A. Skabardonis, "Queue spillovers in city street networks with signal-controlled intersections," in Proceedings of the 89th Annual Meeting of the Transportation Research Board, Washington, DC, USA, 2010.

[18] Y. Sheffi, Urban Transportation Networks: Equilibrium Analysis with Mathematical Programming Methods, Prentice-Hall, Englewood Cliffs, NJ, USA, 1985. 


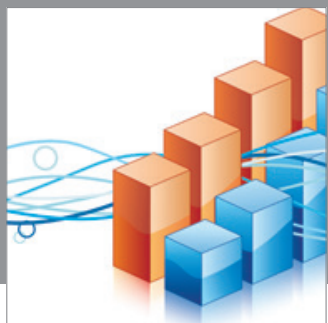

Advances in

Operations Research

mansans

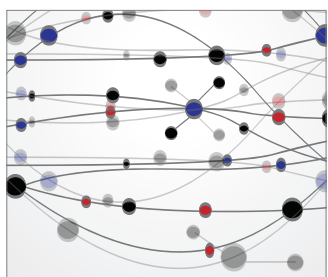

The Scientific World Journal
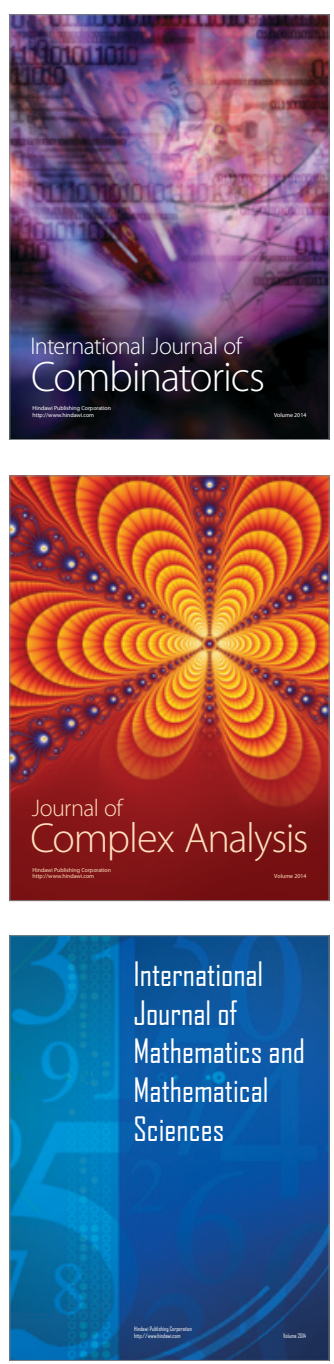
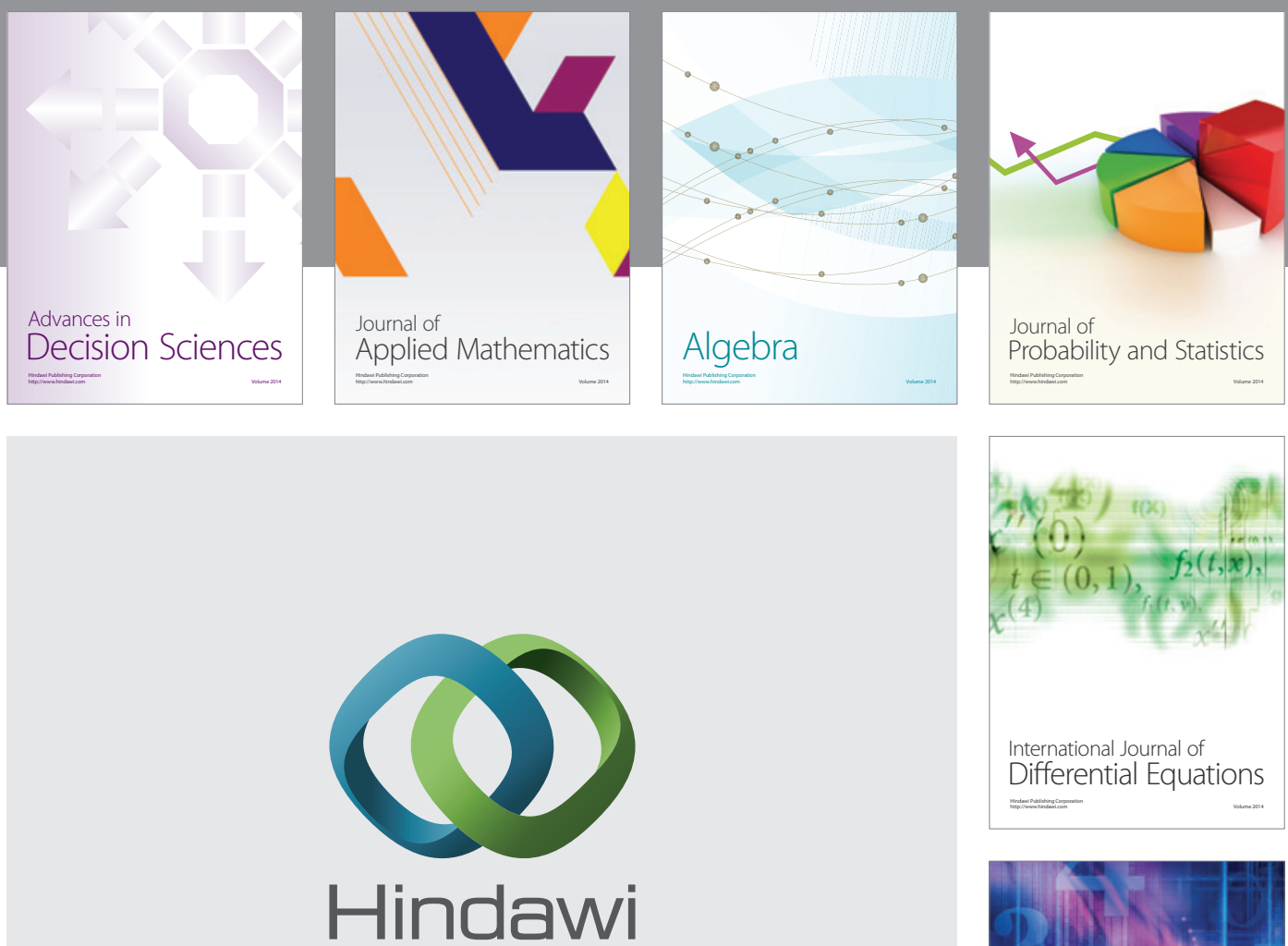

Submit your manuscripts at http://www.hindawi.com
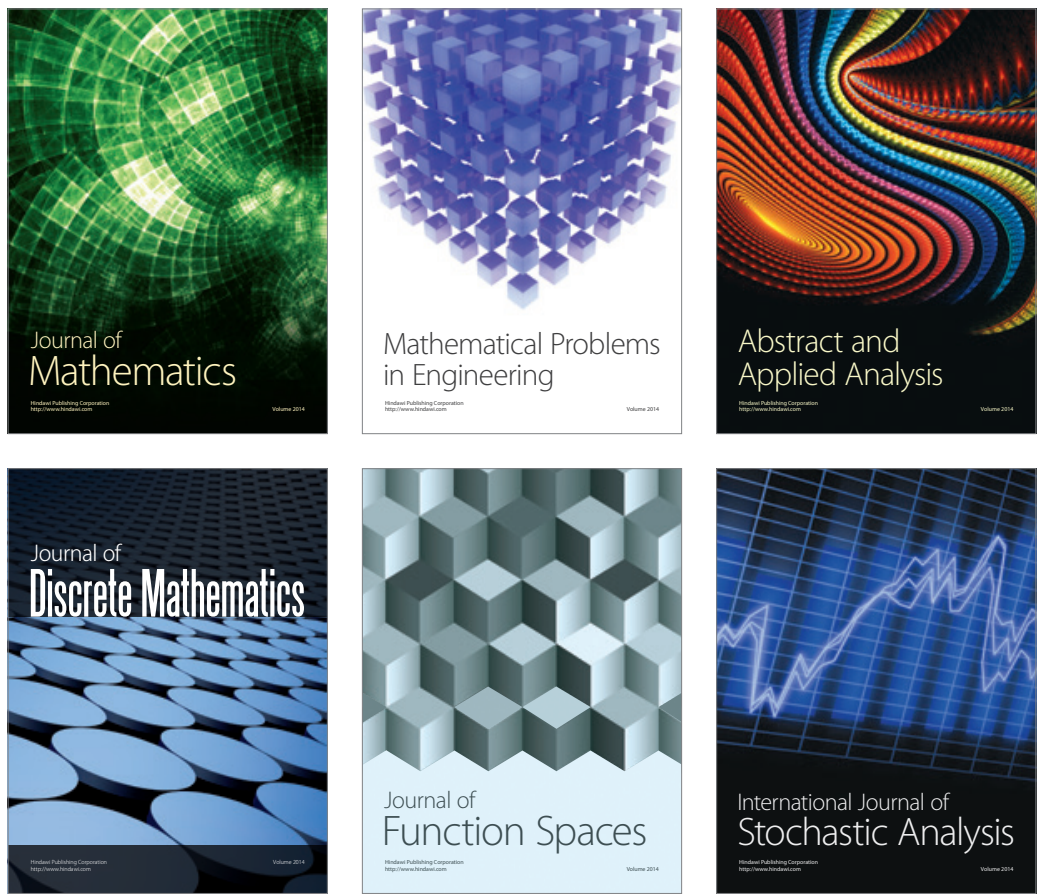

Journal of

Function Spaces

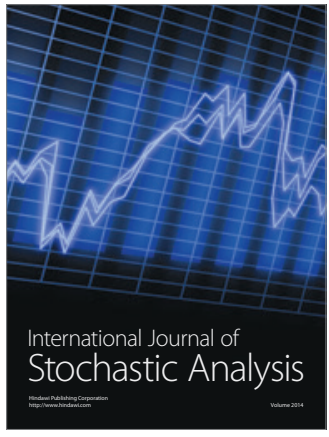

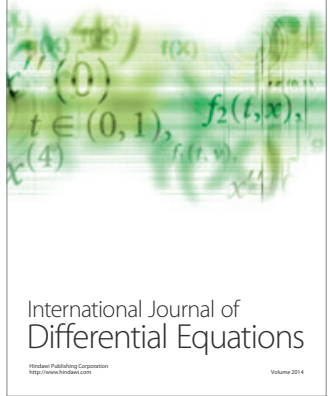
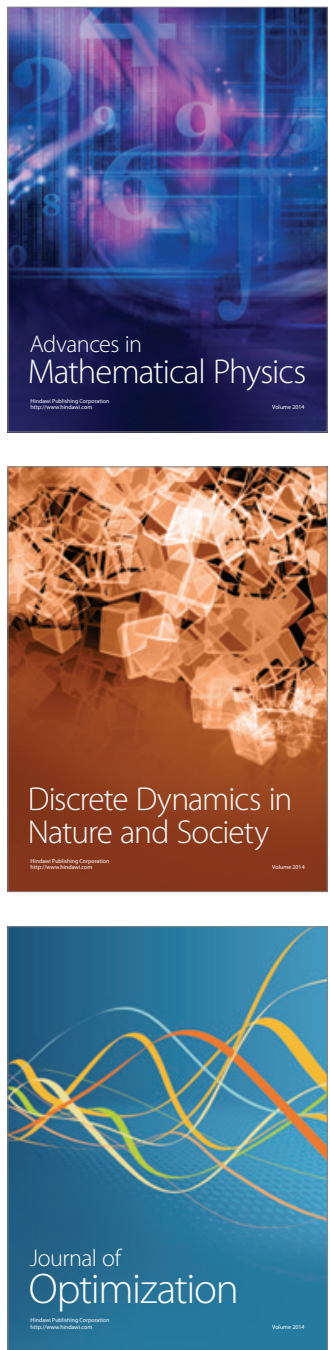